\title{
Successful Treatment of a Ruptured Spontaneous Dissecting Coronary Artery Pseudoaneurysm with a Covered Stent in a Patient with Cardiac Tamponade
}

\author{
Hiroaki Kawano ${ }^{1,2}$, Yuji Matsumoto ${ }^{1,3}$, Osami Satoh ${ }^{1,4}$, Shuji Arakawa ${ }^{1,2}$, \\ Motonobu Hayano ${ }^{1,3}$, Hiroyuki Suyama ${ }^{5}$ and Koji Maemura ${ }^{2}$
}

\begin{abstract}
The rupture of spontaneous dissecting coronary artery pseudoaneurysms is rare, and no standard therapy has yet been established for this condition. This report describes a case of a ruptured spontaneous dissecting coronary artery pseudoaneurysm in a patient with cardiac tamponade that was successfully treated with emergent implantation of a covered stent.
\end{abstract}

Key words: coronary artery aneurysm, intervention, stent, cardiac tamponade

(Intern Med 53: 1067-1070, 2014)

(DOI: 10.2169/internalmedicine.53.1322)

\begin{abstract}
Introduction
Spontaneous coronary artery dissection is defined as dissection in the media without percutaneous coronary intervention (PCI); it represents a rare cause of acute myocardial ischemia and sudden death (1). However, no reports have described the successful treatment of ruptured pseudoaneurysms associated with spontaneous coronary artery dissection.

A covered coronary stent is a new tool for treating coronary artery aneurysms, coronary perforation or rupture due to PCI and represents a less invasive strategy than conventional surgical repair for the treatment of these conditions (2).

The present report describes the case of a patient with cardiogenic shock due to cardiac tamponade related to a ruptured spontaneous dissecting coronary artery pseudoaneurysm. The patient was successfully treated with emergent implantation of a covered stent.
\end{abstract}

Case Report
A 76 -year-old man was referred to our hospital due to
chest discomfort and T wave inversion in leads V2 to V6 on
an electrocardiogram (ECG). A physical examination per-
formed on admission revealed a blood pressure (BP) of 105/
55 mmHg, a pulse of 84 beats/min and a body temperature
of $36.9^{\circ} \mathrm{C}$. A chest X-ray was normal, and transthoracic
echocardiography (TTE) demonstrated a normal left ventri-
cle (LV) systolic function without asynergy.
Laboratory testing showed a white blood cell count of
9,390/mm ${ }^{3}$, a hemoglobin level of 10.1 g/dL, a blood urea
nitrogen level of 18.6 mg/dL, a creatinine level of 0.6 mg/
dL, an aspartate aminotransferase level of 29 IU/L, an
alanine aminotransferase level of $25 \mathrm{IU} / \mathrm{L}$, a lactate dehydro-
genase level of 186 IU/L, a creatine kinase (CK) level of 12
IU/L, a C-reactive protein level of 5.58 mg/dL and a brain
natriuretic peptide level of 83.1 pg/mL. The troponin I level
was within the normal limits, although the serum immuno-
globulin (Ig) E level was extremely high (5,080 IU/L) with
eosinophilia (maximum number, $3,128 / \mathrm{mm}^{3}$ ).
Multidetector computed tomography (MDCT) showed an

\footnotetext{
${ }^{1}$ Department of Cardiology, Nagasaki Rosai Hospital, Japan, ${ }^{2}$ Department of Cardiovascular Medicine, Nagasaki University Graduate School of Biomedical Sciences, Japan, ${ }^{3}$ Department of Cardiology, Sasebo Municipal General Hospital, Japan, ${ }^{4}$ Department of Cardiology, The Japanese Red Cross Nagasaki Genbaku Hospital, Japan and ${ }^{5}$ Suyama Clinic, Japan

Received for publication July 8, 2013; Accepted for publication December 15, 2013

Correspondence to Dr. Hiroaki Kawano, hkawano@nagasaki-u.ac.jp
} 

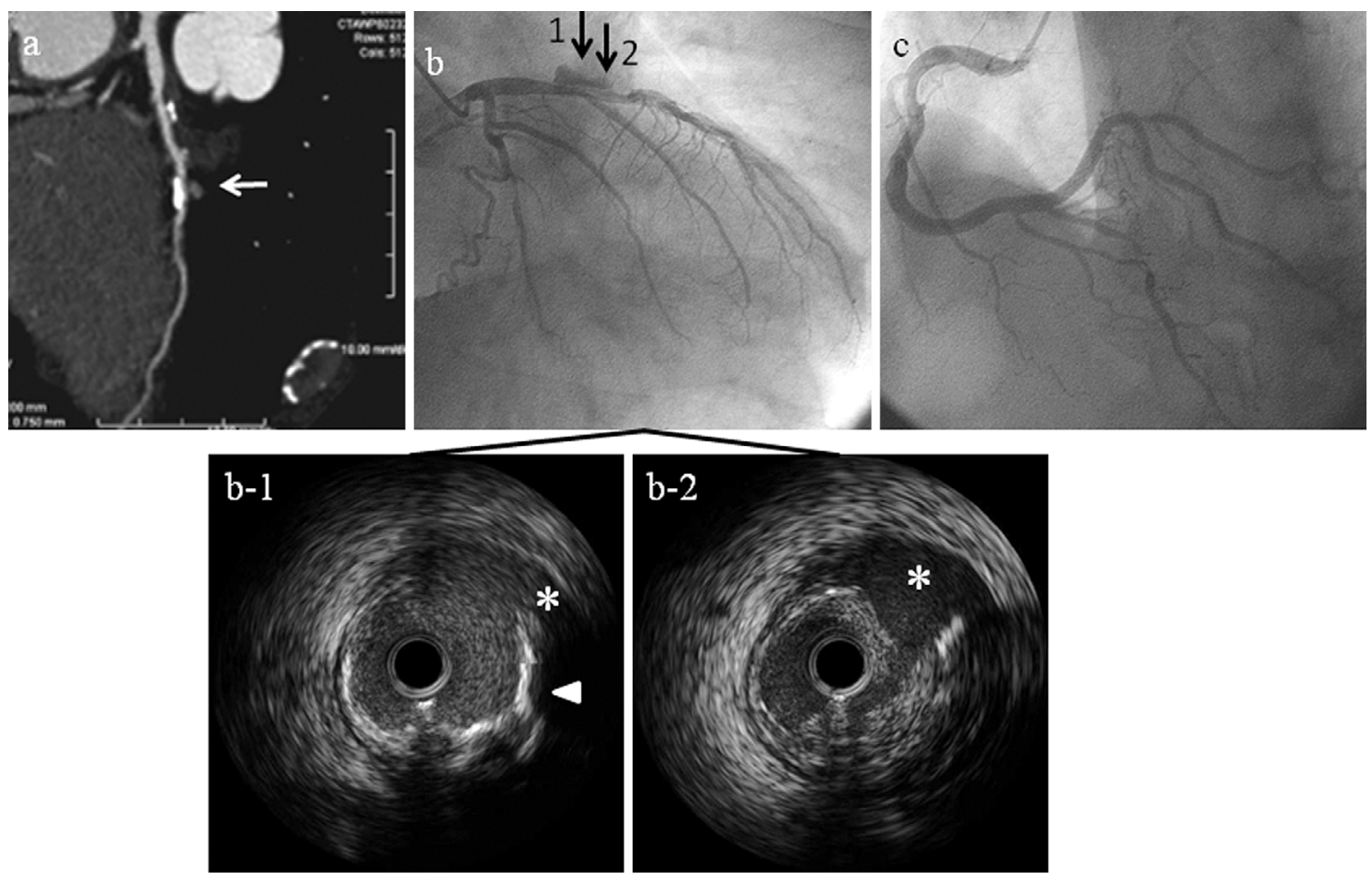

Figure 1. Multidetector computed tomography (a), coronary angiography (b, c) and intravascular ultrasound (b-1, b-2) examinations revealed a dissecting coronary aneurysm (b-1 is an intravascular ultrasound examination image of the arrow \#1 section, and b-2 is an image of the arrow \#2 section) in segment 6 of the left anterior descending artery with calcification (arrowhead). *: pseudolumen

aneurysm with calcification in segment 7 of the left anterior descending coronary artery (LAD) (Fig. 1a). Coronary angiography (CAG) demonstrated $50 \%$ stenosis with a coronary artery aneurysm and dissection in segment 7 of the LAD near the diagonal branch (Fig. 1b, c). An intravascular ultrasound (IVUS) examination using an Atlantis SR pro 2 coronary imaging catheter (Boston Scientific, Boston, USA) showed a coronary artery aneurysm with a flap and pseudolumen in segments 6 and 7 (Fig. 1b-1, b-2).

The patient was treated with aspirin to inhibit thrombus formation in the aneurysm; however, the coronary aneurysm and stenosis were not considered to be large or severe enough, respectively, to require coronary intervention. An evaluation of the elevated $\operatorname{IgE}$ levels via $\operatorname{IgE}$ radioallergosorbent testing was positive for Candida $(3.41 \mathrm{UA} / \mathrm{mL})$, Aspergillus (1.50 UA/mL) and Alternaria (0.72 UA/mL). No evidence of parasitic infection, Wegener's granulomatosis or Churg-Strauss syndrome was noted.

Approximately two weeks after admission, the patient's BP fluctuated and became transiently depressed without symptoms. However, it thereafter returned to a normal level, and TTE showed no abnormal findings, including apparent pericardial effusion. Two days later, the patient developed sudden onset of chest discomfort and hypotension with a BP of 60/40 mmHg. A chest X-ray (Fig. 2a), ECG (Fig. 2b), TTE (Fig. 2c) and chest CT (Fig. 2d) indicated cardiogenic shock due to cardiac tamponade. Following pericardiocentesis and the drainage of blood with a transfusion of lowmolecular-weight dextran and the intravenous infusion of dopamine (5 $\mu \mathrm{g} / \mathrm{kg} / \mathrm{min})$, emergent CAG was performed, which showed leakage of contrast from the ruptured dissecting pseudoaneurysm in segments 6 and 7 without stenosis or occlusion of the coronary artery (Fig. 3a).

Percutaneous covered stent implantation was performed to stop the bleeding. A 7-Fr MAC1 CLS3.5 left guide catheter (Boston Scientific) was engaged in the left coronary artery. IVUS was performed to assess the size of the vessel and determine the stent size. The IVUS examination in addition to coronary angiography revealed a 20 -mm-length ruptured aneurysm extending from segment 6 to 7 . However, the diameter and plaque volume of the dissecting aneurysm could not be measured because the lesion had ruptured and was oversized, although the plaque volume was small. Both the proximal and distal sites of stent implantation were determined to be areas without dissection. The proximal lumen diameter was $3.3 \times 3.0 \mathrm{~mm}$ with a plaque volume of less than $50 \%$, and the media-to-media diameter was $3.9 \times 3.9 \mathrm{~mm}$. The distal lumen diameter was $2.8 \times 2.1 \mathrm{~mm}$ with a plaque volume of less than $50 \%$, and the media-to-media diameter was $3.8 \times 4.2 \mathrm{~mm}$. Based on these IVUS features, two expanded polytetrafluoroethylene (ePTFE)-covered stents (3.0x $16 \mathrm{~mm}$ ) (JOSTENT GraftMaster, Abbott Vascular, Redwood, USA) were directly deployed at 12 atm after crossing the lesion of the LAD with the aneurysm with a 0.014-inch Runthrough NS guidewire (Terumo, Tokyo, Japan). The stents were then dilated using a Powered Lacross balloon catheter $(3.5 \times 15 \mathrm{~mm})$ (Goodman, Nagoya, Japan) four times at 20 atm. Following stent implantation, IVUS was repeated to 


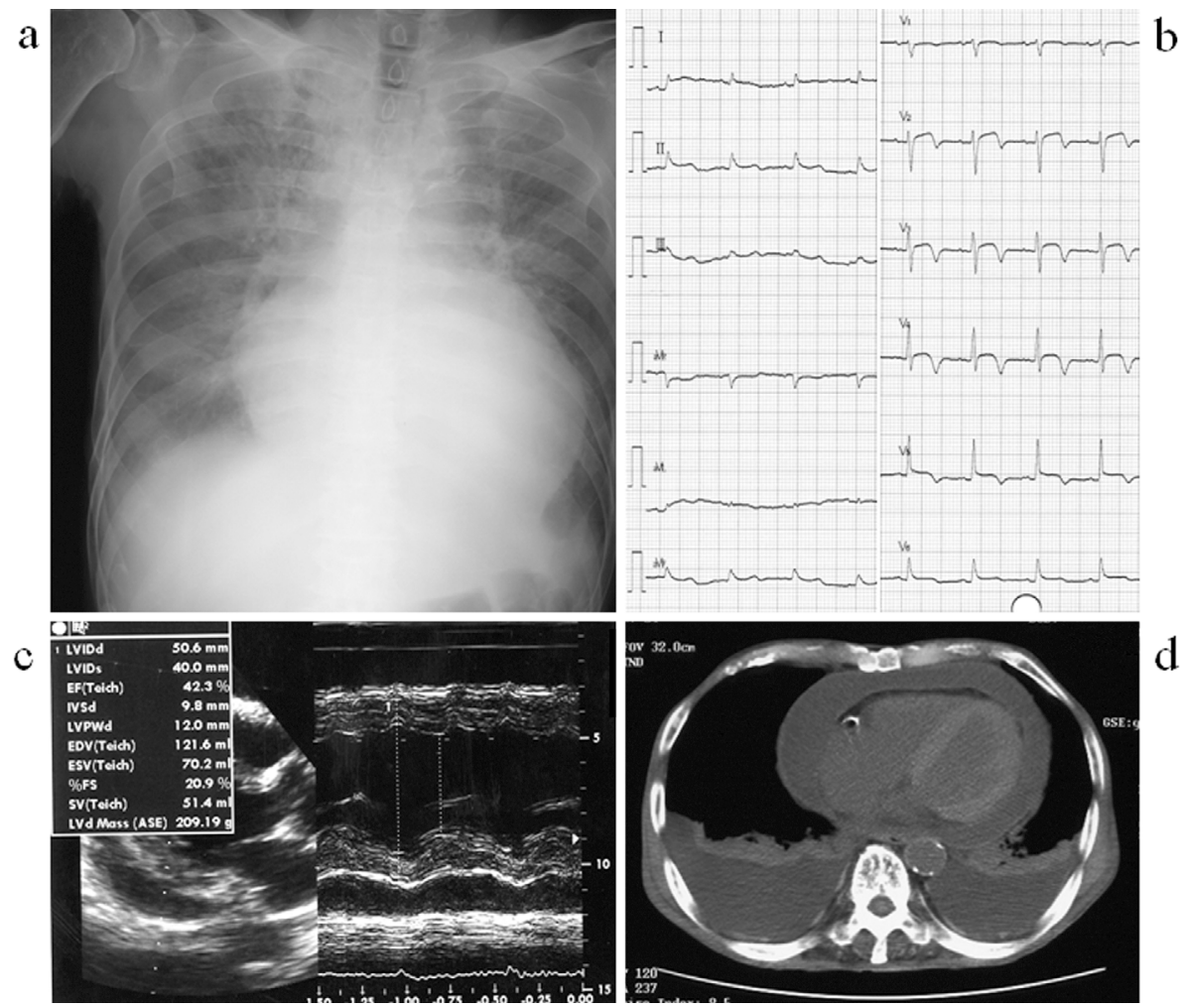

Figure 2. Chest X-ray showed cardiomegaly with pleural effusion (a). Electrocardiography showed low voltage, ST segment elevation in the II, aVF and V2 to V5 leads with terminal $\mathrm{T}$ wave inversion in the V2 to V5 leads (b). Transthoracic echocardiography showed pericardial effusion and mild hypokinesis of the anteroseptal wall of the left ventricle (c), while chest computed tomography showed pericardial and bilateral pleural effusion (d).

confirm stent expansion and the absence of edge dissection or incomplete stent apposition. The final lumen diameter was $3.5 \times 3.5 \mathrm{~mm}$ on IVUS. Unfractionated heparin was administered as an antithrombotic agent during the procedure. After the intervention, the ruptured aneurysm was sealed, and the patient's bleeding stopped approximately two hours after the onset of cardiogenic shock. The CK level was within the normal limits, despite the fact that one diagonal branch was occluded (Fig. 3b).

Following intervention, $100 \mathrm{mg}$ of aspirin and $75 \mathrm{mg}$ of clopidogrel were administered daily. One month after the intervention, chest $\mathrm{x}$-ray showed normal (Fig. 3c), ECG showed normal (Fig. 3d), and CAG showed a normal coronary flow without leakage at the lesion site at which the covered stent had been deployed. The patient did not experience any ischemic episodes over the three-year follow-up period.

\section{Discussion}

Several studies have described the use of ePTFE-covered stents to treat ruptured coronary true aneurysms or coronary artery injury related to PCI (1). One autopsy study described a case of ruptured spontaneous dissection causing cardiac tamponade (3). However, to the best of our knowledge, this is the first report to describe a case of the rupture of a spon- taneous dissecting coronary artery pseudoaneurysm in a patient with cardiac tamponade treated with an ePTFE-covered stent. Performing implantation of a covered stent is easy and can be achieved much more quickly than conventional surgery.

The short- and long-term outcomes after percutaneous implantation of covered stents remain unknown (4). Recent studies have reported delayed endothelialization following PTFE-covered stent implantation when used for the management of coronary aneurysms (5). Therefore, in-stent thrombosis must be prevented after PTFE-covered stent implantation. However, the present patient has not experienced any ischemic complications over the three-year follow-up period under treatment with conventional doses of aspirin and clopidogrel.

The cause of dissecting coronary artery pseudoaneurysm in the present patient appeared to be atherosclerosis, as no evidence of other causes [e.g., Kawasaki disease, Takayasu's disease or connective tissue disorders (Marfan's syndrome, Ehlers-Danlos syndrome)] was apparent. However, the natural history of atherosclerotic aneurysmal coronary artery disease is usually favorable. The present patient exhibited a high C-reactive protein (CRP) level and an extremely high serum IgE level, as well as eosinophilia related to allergies. Eosinophils secrete collagenolytic and basic proteases that can weaken the medial layer of the coronary arteries (6). In- 

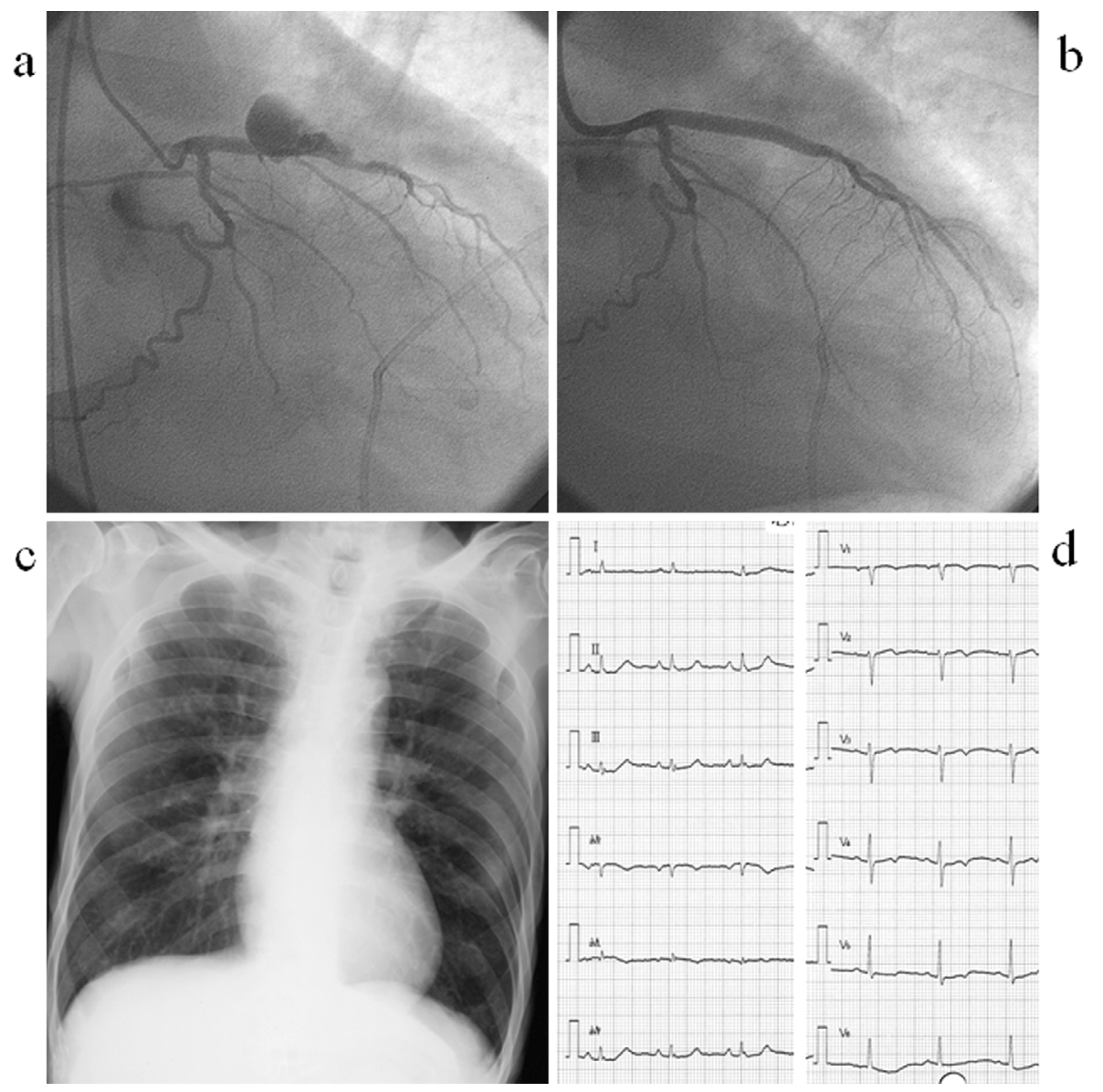

Figure 3. Coronary angiography showed rupture of the coronary artery aneurysm in segment 7 of the left coronary artery (a), resulting in covered stent implantation. Subsequently, the bleeding stopped (b). A chest X-ray (c) and electrocardiography (d) performed approximately one month after the intervention were normal.

deed, a recent study suggested that coronary artery aneurysms and tortuosity are common in patients with hyper-IgE syndrome (7). Taken together, these observations suggest that both hyper IgE and eosinophilia were related to the rupture of the pseudoaneurysm associated with spontaneous coronary artery dissection in the present case.

In conclusion, the use of covered stents should be considered a viable treatment option for the emergent treatment of ruptured spontaneous dissecting coronary artery pseudoaneurysms in patients with cardiac tamponade.

The authors state that they have no Conflict of Interest (COI).

\section{References}

1. Aqel RA, Zoghbi GJ, Iskandrian A. Spontaneous coronary artery dissection, aneurysms, and pseudoaneurysms: a review. Echocar- diography 21: 175-182, 2004.

2. Jamshidi P, Mahmoody K, Erne P. Covered stents: a review. Int J Cardiol 130: 310-318, 2008.

3. Hayes CR, Lewis D. Spontaneous coronary artery dissection of the left circumflex artery causing cardiac tamponade and presenting with atrial fibrillation: a case report and review of the literature. Angiology 58: 630-635, 2007.

4. Briguori C, Sarais C, Sivieri G, Takagi T, Di Mario C, Colombo A. Polytetrafluoroethylene-covered stent and coronary artery aneurysms. Catheter Cardiovasc Interv 55: 326-330, 2002.

5. Takano M, Yamamoto M, Inami S, et al. Delayed endothelialization after polytetrafluoroethylene-covered stent implantation for coronary aneurysm. Circ J 73: 190-193, 2009.

6. Robinowitz M, Virmani R, McAllister HAJ. Spontaneous coronary artery dissection and eosinophilic inflammation: a cause and effect relationship? Am J Med 72: 923-928, 1982.

7. Freeman AF, Avila EM, Shaw PA, et al. Coronary artery abnormalities in Hyper-IgE syndrome. J Clin Immunol 31: 338-345, 2011.

(C) 2014 The Japanese Society of Internal Medicine http://www.naika.or.jp/imonline/index.html 